\title{
À PROPOS DES RÉVOLUTIONS DE COULEUR ET DU SOFT POWER AMÉRICAIN
}

Boris Petric

La Découverte | « Hérodote »

2008/2 $\mathrm{n}^{\circ} 129$ | pages 7 à 20

ISSN 0338-487X

ISBN 9782707154781

Article disponible en ligne à l'adresse :

https://www.cairn.info/revue-herodote-2008-2-page-7.htm

\section{Pour citer cet article :}

Boris Petric, «À propos des révolutions de couleur et du soft power américain 》, Hérodote 2008/2 (n¹29), p. 7-20.

DOI 10.3917/her.129.0007

Distribution électronique Cairn.info pour La Découverte.

(C) La Découverte. Tous droits réservés pour tous pays.

La reproduction ou représentation de cet article, notamment par photocopie, n'est autorisée que dans les limites des conditions générales d'utilisation du site ou, le cas échéant, des conditions générales de la licence souscrite par votre établissement. Toute autre reproduction ou représentation, en tout ou partie, sous quelque forme et de quelque manière que ce soit, est interdite sauf accord préalable et écrit de l'éditeur, en dehors des cas prévus par la législation en vigueur en France. Il est précisé que son stockage dans une base de données est également interdit. 


\section{À propos des révolutions de couleur et du soft power américain}

Boris Petric*

Le 22 mars 2005 à Bichkek, un cavalier kirghize entouré d'une foule déchaînée prend d'assaut le Parlement et l'administration présidentielle. Cette insurrection populaire marque la fin du régime d'Askar Akaev et symbolise une forme de combat politique dans l'espace est-européen et postsoviétique. En effet, ces événements s'inscrivent dans une dynamique politique qui a ensuite communément été appelée « révolutions de couleur » : rose en Géorgie, orange en Ukraine ou des Tulipes au Kirghizstan.

Rapidement, l'interprétation médiatique de ces événements a conduit à un débat idéologique et passionnel tranché : certains y voient le «réveil naturel»et endogène de sociétés qui sortent lentement de l'expérience socialiste, d'autres y perçoivent la main américaine et occidentale, pensant qu'il s'agit d'un complot géopolitique contre la Russie.

Fervent défenseur de l'exportation de la démocratie à l'américaine, Samuel Huntington évoque des vagues successives de démocratisation en Europe. Ainsi, ces révolutions seraient une nouvelle vague dans l'aire postsocialiste après l'effondrement du mur de Berlin (Mc Fall, 2002). Au contraire, pour Gleb Pavlovsky (Pavlovsky, 2006), conseiller politique de Vladimir Poutine, c'est de « renversement », de «coup de force» (perevarot) qu'il est question, visant à saper définitivement l'influence géopolitique de la Russie dans son «étranger proche».

Il faut dépasser ces visions simplistes qui tendent à valoriser l'idée d'une manipulation internationale et à minimiser le rôle des acteurs locaux dans l'évolution

\footnotetext{
* Anthropologue, chargé de recherche au CNRS (IIAC/LAIOS).
} 
de leur société. Ceux-ci ont plus ou moins utilisé le mot «révolution ». Discuter de ce concept pourrait conduire à de très vastes considérations idéologiques. Nous nous bornerons à dire que ces manifestations populaires aspiraient à de grands changements dans l'ordre social et politique.

Dans la plupart des cas, sans parler pour autant d'un renouvellement des élites et d'un changement radical de l'ordre social et politique, on a assisté à une circulation du pouvoir à la tête de l'exécutif. C'est ainsi que, généralement, pour qualifier la nature du changement d'un ordre social et politique en question, le terme révolution est associé à une épithète : «française », "prolétarienne », «bolchevique» « grande», «moderne» (Skocpol, 1979).

La couleur de chacune de ces «révolutions » est celle qui a été portée par certains groupes de manifestants pour se distinguer d'autres groupes partisans d'un statu quo, sinon du gouvernement en place. Ces révolutions ont été - du côté des manifestants comme de celui des forces de l'ordre - pour l'essentiel nonviolentes. L'usage de l'épithète «colorée » perturbe ici le sens que l'on pourrait attribuer à ces mouvements qui ont, de ce fait, été immédiatement lus comme nonviolents, selon la théorie de l'intellectuel américain Gene Sharp dont il sera question à plusieurs reprises.

Généralement, l'instauration d'un nouveau pouvoir politique et social se traduit par une mise à l'écart des anciennes élites (emprisonnement, exil forcé, parfois même exécution). Dans l'espace postsoviétique, les anciens présidents ont été poussés au départ, mais la plupart d'entre eux se sont très vite recyclés dans la logique des nouveaux régimes.

À travers ces révolutions colorées, un certain nombre de questions se posent quant aux fonctionnements du pouvoir politique. Les épisodes géorgien, ukrainien, kirghize peuvent-ils être interprétés comme une modification radicale des principes de légitimité ? Peut-on parler de révolutions démocratiques dans lesquelles émergerait une légitimité populaire ? La naissance d'un système démocratique se réduit-il aux épisodes électoraux ? Comment s'exercent le pouvoir et la souveraineté dans ces jeunes États?

Ces processus politiques s'inscrivent dans une période d'intensification de circulations d'idées - qu'il s'agisse de démocratie ou de celle de personnes, comme les experts de la démocratisation. Ces révolutions sont ici finalement une occasion de mieux comprendre un processus politique plus large, qui est celui de la redéfinition des acteurs politiques dans ces pays depuis la fin de la guerre froide, la chute du mur de Berlin et l'implosion de l'Union soviétique.

Ce numéro d'Hérodote comprend un certain nombre d'articles qui traitent précisément de ces mouvements. Ces articles sont le résultat d'une journée d'études que j'ai organisée en octobre 2005 à l'EHESS, regroupant des chercheurs s'intéressant plus particulièrement aux relations tissées entre « experts de la promotion 
de la démocratie » et «acteurs locaux ». Cela renvoie essentiellement à ce que l'on appelle communément le soft power (Nye, 1990), c'est-à-dire une technique d'influence fondée sur la capacité d'attraction d'un modèle politique. Des ONG américaines sont les principaux acteurs de cette promotion de la démocratie, des droits de l'homme et de la liberté, mais des ONG scandinaves sont également actives ainsi que des fondations allemandes (Dakowska, 2006). Certains de ces acteurs (ONG, fondations, etc.) ne dépendent pas de finances publiques et peuvent être difficilement rattachés à l'action d'un État-nation ou d'un gouvernement (à l'instar de la fondation Open Society du milliardaire George Soros). Cependant, il ne faut pas établir des frontières trop rigides, dans la mesure où ces acteurs circulent, au gré des emplois et des projets, au sein des grandes ONG et des institutions internationales (PNDU, OSCE, Banque mondiale, etc.) afin de diffuser une idéologie commune de la «bonne gouvernance » qui consiste souvent à promouvoir l'économie de marché et la démocratie libérale. Après 1989, les organisations non gouvernementales (ONG), certaines fondations philanthropiques et les organisations internationales sont au cœur de la naissance des nouveaux acteurs sociaux dans l'aire postsocialiste et participent aux rapports de pouvoir au sein de ces sociétés. Et il y aurait d'ailleurs des parallèles intéressants à faire avec les travaux de sciences sociales qui s'intéressent au poids de ces acteurs dans l'évolution des sociétés en voie de développement (Ferguson, 1990). Si l'on se réduit à l'aire postsocialiste, la confrontation des différentes expériences « révolutionnaires colorées » permet de dégager une typologie d'acteurs ${ }^{1}$ inédits qui s'esquisse déjà dans la société serbe et que l'on retrouve dans l'ensemble des pays concernés.

Il y a dans ces révolutions de couleur une similitude dans la temporalité : le scénario révolutionnaire intervient au moment d'élections contestées et dans un contexte où l'État est amené à se désengager de nombreux secteurs d'activités. Le moment électoral devient le théâtre d'une confrontation entre le gouvernement d'un côté et plusieurs acteurs émergents de l'autre : les mouvements étudiants, des coalitions d'ONG, des coalitions d'opposition et des médias privés. C'est sur ces quatre catégories d'acteurs que l'on souhaite particulièrement attirer l'attention des lecteurs.

1. $C f$. tableau ci-après. 
LES ACTEURS LOCAUX

\begin{tabular}{|l|l|l|l|l|}
\hline Pays & $\begin{array}{l}\text { Mouvements } \\
\text { étudiants }\end{array}$ & $\begin{array}{l}\text { Coalition de } \\
\text { l'opposition }\end{array}$ & $\begin{array}{l}\text { Coalition } \\
\text { des ONG }\end{array}$ & $\begin{array}{l}\text { Médias } \\
\text { indépendants }\end{array}$ \\
\hline $\begin{array}{l}\text { Géorgie } \\
\text { Novembre } \\
2003\end{array}$ & $\begin{array}{l}\text { KMARA } \\
\text { Rose }\end{array}$ & $\begin{array}{l}\text { Démocrates } \\
\text { unis }\end{array}$ & $\begin{array}{l}\text { ISFED } \\
\text { International Society } \\
\text { for Free Elections } \\
\text { and Democracy }\end{array}$ & $\begin{array}{l}\text { Roustavi 2 TV } \\
\text { 24 heures }\end{array}$ \\
\hline $\begin{array}{l}\text { Ukraine } \\
\text { Décembre }\end{array}$ & $\begin{array}{l}\text { POR4 } \\
\text { Orange }\end{array}$ & $\begin{array}{l}\text { Notre } \\
\text { Ukraine }\end{array}$ & $\begin{array}{l}\text { CVU } \\
\text { Committee of } \\
\text { Voters of Ukraine }\end{array}$ & $\begin{array}{l}\text { Kanal 5 } \\
\text { Ukrainskaja Pravda } \\
\text { Radio Liberté }\end{array}$ \\
\hline $\begin{array}{l}\text { Kirghizstan } \\
\text { Mars 2005 }\end{array}$ & $\begin{array}{l}\text { KEL-KEL } \\
\text { Tulipes }\end{array}$ & $\begin{array}{l}\text { Mouvement } \\
\text { populaire du } \\
\text { Kirghizstan }\end{array}$ & $\begin{array}{l}\text { KOALITSIA } \\
\text { Coalition for Democracy } \\
\text { and Civil Society }\end{array}$ & $\begin{array}{l}\text { Pas de télévision } \\
\text { Journal MSN } \\
\text { Radio Azatik }\end{array}$ \\
\hline
\end{tabular}

\section{L'ouverture et l'influence étrangère}

S'il est indéniable que l'exaspération populaire prend une consistance et une ampleur particulières dans ces pays qui pour la plupart n'ont pas connu d'alternance politique depuis la chute du mur de Berlin, un certain nombre de conditions devraient être réunies pour organiser la conquête du pouvoir. Les élites de ces régimes sont généralement accusées d'avoir bénéficié de la privatisation de certains moyens de production, ce qui a provoqué, en grande partie, un accroissement des inégalités. Cela n'a pas échappé à une opinion publique naissante qui a pris rapidement conscience des problèmes. Cependant, les différentes formes de mécontentement ne peuvent déboucher sur de nouveaux processus politiques que si elles sont canalisées par un ensemble d'acteurs inédits et identifiables qui structurent la contestation, en mettant en scène de nouvelles sources de légitimité dans un espace public à construire. C'est pourquoi ces révolutions colorées ne peuvent être analysées uniquement en termes d'exaspération populaire et de généralisation de la pauvreté. De nombreux autres pays du monde où sévissent accroissement des inégalités et corruption ne connaissent pas pour autant ce genre de scénarios.

Dès le début des années 1990, de profonds changements marquent l'affirmation de nouveaux protagonistes (soutenus par des organisations internationales ou des ONG) qui jouent un rôle déterminant dans l'organisation de ces révolutions. L'appel international à l'émergence d'une véritable société civile qui aurait été en 
sommeil à l'époque soviétique a suscité des critiques en relation avec la banalisation de cette notion (Hann, 2002). Sous couvert d'une assistance technique, la référence à la société civile est devenue peu à peu un leitmotiv trop normatif pour intervenir dans la vie politique de ces pays (Kilhani, 2001). Elle s'est imposée comme une norme internationale pour définir une société qui se caractérise par l'existence d'un tissu d'ONG qui s'organisent et collaborent pour affronter un gouvernement qui tend à se penser comme indissociable de l'État. On peut parler d'une «ongénéisation » des sociétés, ce qui s'inscrit justement dans un processus mondialisé de modification radicale du rôle de l'État (Appadurai, 2002); or, la société civile ne peut pas se penser uniquement en termes d'opposition et de rapport univoque à l'État. Cependant, pour l'univers postsocialiste (Burawoy, 1999), les ONG locales - dont la prolifération témoigne du renforcement de rapports transnationaux qui modifient les relations sociales et politiques - constituent probablement l'acteur le plus significatif et marquent le sceau d'un changement de la nature du pouvoir dans ces sociétés. Dans ces pays, les ONG ne peuvent être réduites uniquement à une influence extérieure. Elles illustrent l'émergence de nouvelles institutions sociales dont la singularité est de bénéficier de ressources matérielles ou immatérielles captées à l'extérieur de la société pour participer à la vie sociale ou politique au niveau local.

Ces sociétés, qui sont fortement marquées par un désengagement de l'État, sont contraintes de passer d'un système social de planification vers une «vie sociale de projet» (Sampson, 1996) où les ONG doivent être au cœur de l'initiative sociale. Nombre d'entre elles s'inscrivent dans une logique politique globale qui vise à contester le poids de l'État dans les affaires de la Cité. Ces ONG ont proliféré, dès le début des années 1990, grâce aux multiples soutiens financiers de fondations étrangères et de nombreuses organisations internationales.

\section{L'émergence de nouveaux acteurs}

Ces révolutions colorées s'inscrivent dans une histoire et celle-ci nécessite de revenir à une généalogie historique en suivant, au gré des situations politiques, la circulation des idées et des hommes qui sont au cœur des changements. D'ailleurs, la métaphore de la vague ou de la contamination utilisée par de nombreux médias ne permet pas forcément de comprendre l'émergence de ces mouvements sociaux. En revanche, les parcours des acteurs, les idées qui circulent dans les formations de la promotion de la démocratie sont des outils d'analyse pertinents.

Parmi ces acteurs, les mouvements de jeunesse occupent une place de premier choix, Otpor en Serbie, Kmara en Géorgie, Pora en Ukraine ou Kel-Kel au Kirghizstan. Ils s'inspirent des mouvements de résistance non-violents et ont 
bénéficié de nombreuses formations pour rationaliser leur lutte dès leur création. Ils se sont inspirés des idées du politologue américain Gene Sharp, diffusées par l'Albert Einstein Institute ${ }^{2}$, mais aussi de Freedom House. Ces mouvements ont une dimension transnationale car ces jeunes élites tissent, au gré des sessions de formation et des expériences - dans leur pays ou à l'étranger -, des liens étroits avec les acteurs de la promotion de la démocratie.

\section{Otpor, le mouvement étudiant serbe : Résistance contre Milosevic}

On limite généralement les révolutions de couleur à l'espace postsoviétique (Géorgie, Ukraine, Kirghizstan). Les acteurs transnationaux qui ont joué un rôle de premier plan dans ces événements sont déjà présents dans certains pays d'Europe de l'Est. Certains mouvements étudiants ont joué un rôle déterminant dans les mouvements collectifs qui affectent l'Europe de l'Est après la chute du mur de Berlin. On peut citer notamment le mouvement slovaque OK'98 mais c'est certainement le mouvement étudiant serbe Otpor qui est le plus connu. Otpor joue un rôle décisif au moment où le pouvoir de Slobodan Milosevic tombe en Serbie. Cette mobilisation intervient dans un contexte particulier: elle fait suite aux bombardements sur la Serbie par l'OTAN (1999) et à la crise du Kosovo. Il ne faut pas y voir seulement un modèle de référence intellectuelle dont se seraient vaguement inspirés les différents pays. Il y a une continuité dans l'émergence d'institutions et d'acteurs inédits. Après l'échec électoral de 1996, une nouvelle dynamique s'enclenche dans l'opposition, qui est divisée. La stratégie consiste alors à créer une large coalition politique, mais aussi civile, avec des mouvements sociaux face au pouvoir en place. C'est ainsi qu'émergent des acteurs exprimant cette nouvelle cohésion. Plus tard, certains acteurs serbes ont participé à la formation de leurs homologues dans l'espace postsoviétique à travers de nombreux séminaires, summer-camps et workshops. Au nombre de quatre, ils ont tous développé des liens structurels avec des ONG ou des fondations internationales de la promotion de la démocratie.

Ces mouvements se caractérisent par une intense production culturelle qui témoigne aussi que l'on peut difficilement les réduire à un instrument de manipulation produit par une puissance étrangère. Les révolutions ont vu l'émergence de communications mêlant politique et références culturelles à travers des vidéoclips, des films satiriques ou encore des chansons et des livres. Des formes inédites de mobilisation ${ }^{3}$ qui déstabilisent les forces de l'ordre sont apparues. Dans ce

2. www.aeinstein.org.

3. Des flash-mob ont notamment eu lieu en Ouzbékistan devant l'ambassade des USA. Il s'agit de réunir un maximum de personnes devant un lieu public au cours d'un moment bref 
Otpor (Résistance) est le mouvement de jeunesse qui émerge en 1998 après un conflit sur la remise en cause du statut d'autonomie de l'université. Son symbole est un poing noir levé incarnant la «résistance». Un slogan marque l'opinion, «Gotov je », signifiant à la fois que Milosevic doit partir (il est fini) et qu' une alternance est possible (il est temps). Ce mouvement organise avec la coalition d'opposition DOS la marche sur Belgrade, encadrant les manifestations et la foule qui prend d'assaut le Parlement serbe le 4 octobre 2000. Ce mouvement de jeunesse, issu de la résistance non-violente, donnera une vision unitaire à une contestation populaire très variée et hétéroclite. Il s'est éteint progressivement et aujourd'hui certains leaders, dont Srdjan Popovic, ont créé une société de conseil, «Center for Non Violent Resistance », exportant les techniques de résistance civile.

Le CESID est une coalition d'ONG locales serbes (Center for Free Elections and Democracy) créée en 1997. Il occupe une place déterminante dans l'observation des élections et la production de résultats et de sondages alternatifs face au régime Milosevic au moment des élections du mois d'octobre 2000. Il observe régulièrement les élections en Serbie mais aussi dans d'autres pays de l'Est et en ex-URSS dans le cadre de sa participation au réseau transnational ENEMO. Le CESID en a dirigé la présidence en 2000-2001.

DOS (Democratic Opposition of Serbia) est une coalition regroupant 18 partis politiques d'opposition. L'idée d'une alliance émerge suite à l'échec de l'opposition aux élections de 1996, dû en partie à son éclatement face au Parti socialiste très structuré de Slobodan Milosevic. Elle est formée en 2000 au moment des élections présidentielles fédérales, et animée par Vojslav Kostunica et Zoran Djindjic en 2000. Cette forme de coalition inédite intervient après de longues années où l'opposition n'arrive pas à se structurer face au pouvoir de Milosevic et éclatera rapidement après.

Radio/TV B92 est un média indépendant fondé par Veran Matic. Elle fait partie des multiples radios libres locales qui commencent à émettre en 1989 et sera interdite d'émission à plusieurs reprises sous Milosevic. En septembre 2000, elle obtient l'autorisation d'émettre comme chaîne de télévision pour le grand Belgrade et la Voïvodine et diffuse les journaux télévisés des grands médias occidentaux. Avec certains journaux récents (Danas, etc.), ces nouveaux médias ont permis une médiatisation du mouvement de contestation sur l'ensemble du territoire, soit par la radiodiffusion soit par internet pour éviter la censure. Veran Matic a été président de l'ANEM (Association des médias électroniques indépendants) et est l'un des fondateurs d'une coalition mondiale de médias indépendants (Free 2000).

pour diffuser des slogans protestataires. La manifestation se disperse rapidement pour éviter la répression policière. 
contexte, la diffusion de l'information par les nouvelles technologies à l'aide des téléphones mobiles ou de l'internet modifie le rôle des frontières, restreint le pouvoir de contrôle de l'État et forme une nouvelle sphère publique. De plus, la profusion d'images touche à l'imaginaire profond de ces sociétés : les séries télévisées matérialisant la corruption du pouvoir, des jeux vidéo proposant de faire tomber une dictature, des films satiriques caricaturant le gouvernement ont contribué à remettre en cause l'autorité des pouvoirs en place et à imaginer un autre possible.

\section{$\underline{\text { L'exportation de la démocratie par les États-Unis }}$}

L'intensification des flux d'idées se matérialise dans les années 1990 par la généralisation de la référence à la démocratie. De très nombreux programmes de développement et de démocratisation sont portés par des acteurs en majorité américains (Robinson, 1996). La promotion de la démocratie est au cœur de la politique extérieure américaine, marquée par le fameux discours de Westminster prononcé par Ronald Reagan en 1983. Progressivement, cette idée devient un programme avec des institutions et des protagonistes identifiables. Une nouvelle élite, les «democracy makers » (Guilhot, 2006), intervient au sein d'institutions américaines - le National Endowement for Democracy (NED) -, puis internationales (PNUD, OSCE, Banque mondiale), mais aussi de fondations privées comme la Fondation Soros, pour exporter la démocratie dans le monde. Le NED, institution publique américaine, finance, entre autres, les fondations des Partis démocrate (National Democratic Institute) et républicain (International Republican Institute), qui ont été très actives dans la transition de ces pays, notamment dans la formation des coalitions d'ONG et de partis politiques. Compte tenu de la mondialisation des phénomènes politiques, il apparaît notamment indispensable d'engager un dialogue entre des spécialistes de l'espace postsocialiste et des chercheurs ayant travaillé sur ces institutions de la promotion de la démocratie. Qu'elle soit matérielle ou idéelle, la mondialisation des échanges nous amène à porter notre attention sur l'émergence de logiques et de réseaux transnationaux qui nécessitent des collaborations croisées avec des non-spécialistes de l'espace postsocialiste.

L'entretien avec le politiste Jacques Sémelin nous apporte un éclairage indispensable sur les racines intellectuelles dont se réclament notamment les mouvements étudiants Otpor, Kmara, Pora et Kel-Kel. Sa connaissance des mouvements non-violents, théorisée par le philosophe Gene Sharp, et de l'histoire de la radio Free Europe nous permet de comprendre les fondements intellectuels de ces mouvements locaux, et de saisir la place stratégique prise par la médiatisation 
des émotions dans les scenarios révolutionnaires. Enfin, le compte rendu de Mayeul Kauffman sur l'opuscule de Gene Sharp est ici précieux pour une lecture transversale de ces mouvements.

En effet, il apparaît de plus en plus difficile d'appréhender ces sociétés suivant des catégories traditionnelles : politique nationale, État, institutions, partis. Des acteurs transnationaux évoluent dans ces nouveaux espaces de la mondialisation - certains y voient la naissance d'une « internationale de la démocratie».

Si la notion de «révolution» est immédiatement prise dans un débat idéologique, il faut cependant tenir compte de ce que disent les acteurs au moment des faits pour anticiper sur les événements politiques futurs, au cours desquels les citoyens réinterprètent (Koselleck, 1994) la mobilisation collective du temps révolutionnaire. Ces nouvelles élites ont des valeurs communes, des codes et fréquentent de nouveaux lieux de pouvoir non seulement à l'intérieur de leur propre société mais aussi dans leur espace régional. Cet enchevêtrement entre local et global demande que les chercheurs s'investissent dans ces nouveaux champs d'études.

Les coalitions d'ONG constituent une de ces nouvelles formes d'institutions qui participent à la construction de la légitimité du pouvoir en s'associant à l'observation électorale, devenue un élément incontournable de la vie politique de ces pays.

Si ces révolutions ont occupé une très forte place médiatique en Europe occidentale, l'émotion qu'elles ont provoquée est autant à prendre en considération dans l'analyse des phénomènes politiques. Des images circulent, sont récupérées, appropriées dans des situations locales différentes. Cependant, l'idée de contagion, de vague ne suffit pas à apprécier l'ensemble du processus, qui n'est pas réductible aux symboles télévisuels. Les formations dispensées dans les milliers de séminaires, de summer-camps ou de workshops proposés par les ONG, fondations ou institutions internationales permettent de diffuser de nouvelles conceptions de la société. Appartenir à cette nouvelle sphère internationale, parler l'anglais, voyager deviennent des critères de distinction sociale.

Dans ce contexte, la télévision et les médias privés jouent un rôle de premier plan, comme en Géorgie et en Ukraine, avec l'émergence de nouvelles chaînes de télévision. Ces dernières contribuent à la constitution d'un nouvel espace public et sont liées notamment à l'existence de liens internationaux et transnationaux avec des fondations et des médias étrangers. 


\section{Caractéristiques géopolitiques communes à ces « révolutions »}

Ces changements ont également une portée géopolitique. L'ouverture de l'Eurasie postsocialiste a provoqué une redéfinition de la souveraineté et de la légitimité des pays de ce très vaste ensemble géopolitique. La vie politique intérieure s'est émancipée de l'ancienne tutelle moscovite tout en faisant face à de nouvelles contraintes de plus en plus pesantes, qui ne se limitent pas aux influences des grandes puissances étrangères et voient le renforcement du rôle des organisations internationales ou régionales. La Géorgie, l'Ukraine et le Kirghizstan ont pour caractéristique commune de se trouver aux marges de l'ancien espace soviétique. Ces pays de confins, de marches sont tiraillés par différentes influences impactant directement leur vie politique intérieure. Alors que l'on pensait la guerre froide définitivement révolue, ces révolutions semblent être le reflet de rivalités notamment entre Américains et Russes et caractérisent la montée en puissance des organisations internationales (ONU, FMI, Banque mondiale) ou régionales qui ne laissent plus le monopole de l'influence à l'Etat (Union européenne, Groupe de Shanghai, OSCE).

Ces révolutions peuvent être analysées aussi comme un théâtre de confrontation de différentes techniques d'influence. Désormais, dans l'Eurasie postsocialiste, tout scrutin national comporte un enjeu international, opposant des forces souhaitant peser sur les réalités sociales de ces pays. Pour ce faire, certaines s'appuient sur des référents extérieurs pour revendiquer une nouvelle légitimité et asseoir leur pouvoir. On constate néanmoins, après ces révolutions, l'utilisation de la promotion de la démocratie comme moyen d'accéder au pouvoir, sans avoir réellement l'intention de changer les règles du jeu.

Dans un premier temps, les analyses de ces événements ont été marquées par une polarisation simpliste : côté russe, la théorie du complot, consistant à voir dans ces révolutions essentiellement la main américaine ; de l'autre, la minimisation ostensible du rôle des ONG et des autres acteurs occidentaux dans les changements en cours. Il n'en reste pas moins que l'on peut d'ores et déjà établir certaines analyses sur ces révolutions colorées. Elles se sont produites dans des pays où, depuis 1991, des hommes politiques de l'époque soviétique incarnaient le pouvoir: Edouard Chevarnadze en Géorgie, Leonid Koutchma en Ukraine et Askar Akaev au Kirghizstan, qui avaient d'ailleurs entamé des réformes beaucoup plus importantes que dans les pays voisins. Elles ont été précédées par un important désengagement de l'État dans de nombreux secteurs (économie, social, éducation, médias), laissant la place à l'émergence de nouveaux acteurs. La personnalisation du pouvoir fait place au renforcement du rôle du Parlement. Il apparaît que la politique étrangère schizophrénique adoptée par les présidents misant sur un jeu de l'équilibre entre Américains, Européens et Russes pour gagner en souveraineté a 
fini par les mettre hors jeu. Au bout d'un certain temps, ces stratégies d'équilibristes mettent au jour des contradictions difficilement tenables. La transition politique de ces pays s'inscrit dans une montée en puissance des organisations internationales, qui sont désormais des éléments essentiels de l'exercice du pouvoir et participent à la construction de la légitimité d'un espace politique.

Ces révolutions sont la concrétisation du modèle de valorisation de la démocratie au plan mondial. Certains avaient d'ailleurs parlé de la «fin de l'histoire» au moment de l'effondrement du mur de Berlin (Fukuyama, 1992), pensant assister au triomphe d'un seul modèle politique et économique : la démocratie et l'économie de marché. Cependant, la multiplicité des situations montre des réappropriations inattendues d'acteurs locaux à la conquête du pouvoir et des réactions divergentes qui donnent tort à $\mathrm{F}$. Fukuyama, même si la référence à la démocratie et à la liberté sature l'idéologie politique américaine alors que ce pays mène une guerre en Irak et en Afghanistan. De plus, après les révolutions, les évolutions politiques prennent en Ukraine, en Géorgie et au Kirghizstan des tournures inattendues et des rivalités sont apparues rapidement entre les leaders, ce qui d'ailleurs amène à soumettre à l'examen critique les premières impressions après les changements de pouvoir ${ }^{4}$. Une fois le pouvoir conquis, la référence à la démocratie n'est souvent plus une priorité et les mêmes logiques de pouvoir s'instaurent. Les programmes de la promotion de la démocratie sont donc aussi souvent l'objet d'une manipulation de la part d'un groupe local qui cherche à conquérir le pouvoir.

Les réactions suscitées ailleurs (Azerbaïdjan, Biélorussie, Russie, Ouzbékistan) nécessitent l'analyse d'un type de modèles politiques qui se veulent ouvertement une alternative à l'assistance démocratique proposée par les Occidentaux.

Les conditions d'instauration des nouveaux systèmes politiques, qui se réfèrent à la démocratie, amènent à s'interroger sur le politique dans un contexte où la présence internationale (UE, ONU mais aussi les ONG) redéfinit les lieux de pouvoir. Les acteurs internationaux et transnationaux participent au jeu de pouvoir local et contribuent à construire et déconstruire la légitimité d'un espace politique. Où se situe le pouvoir dans ces sociétés ? N'a-t-on pas assisté à des déplacements importants de l'exercice de celui-ci ? Peut-on réduire le corps politique aux seuls

4. On remarque notamment qu'il y a systématiquement un homme, une femme à la tête des coalitions d'opposition dans les différents pays, comme s'il fallait respecter un "gender balance » cher aux organisations internationales. Rapidement, le rôle des femmes passe au second plan après la conquête du pouvoir. Roza Otumbaeva au Kirghizstan, Ioulia Timochenko en Ukraine ont été évincées. En Géorgie, Nino Bourdjanadze est présidente du Parlement. Une autre femme, Salomé Zourabichvili, ministre des Affaires étrangères, a quitté le gouvernement de Saakashvili. Elles ont toutes dénoncé les pratiques politiques de leurs éphémères compagnons de route. 
citoyens et hommes politiques et quel statut doit-on donner aux divers experts qui influencent la réalité politique de ces pays? N'y a-t-il pas tout un ensemble d'acteurs qui implique de réévaluer les frontières classiques du politique?

\section{La stratégie de la Russie}

Il faut également tenir compte des pays dans lesquels le scénario révolutionnaire était attendu (Azerbaïdjan, Ouzbékistan ou Biélorussie) mais n'a pas eu lieu. Le succès des oppositions aux élections entraîne la naissance d'une réaction des pouvoirs en place, avec de nouveaux soutiens. Akaev avait tenté de lancer, après le référendum de 2002, une coalition d'ONG, «Associatsia», pour contrecarrer l'influence de Koalitsia et de son très médiatique président Edil Baïsalov. Au cours de la Révolution orange, on a beaucoup parlé des «temniki» (messages), ces instructions envoyées par l'administration présidentielle aux journalistes afin d'orienter l'analyse des informations en Ukraine. On a aussi évoqué le rôle des "politeknolog », ces techniciens de la politique russe chargés d'aider les pouvoirs en place à répondre au combat. Enfin, il faut signaler la réaction de certains pays mettant fin à l'activité d'organisations étrangères. La fondation Soros a fermé ses portes en Russie en 2000 puis en Ouzbékistan en 2003. L’ONG américaine Freedom House a également été expulsée d'Ouzbékistan et de nombreux pays mettent en place une nouvelle législation sur l'activité des ONG, comme la Russie, l'Ouzbékistan mais aussi, et cela est plus surprenant, le Kirghizstan. C'est pour cette raison que la riposte russe doit attirer notre attention pour comprendre l'émergence d'une nouvelle conception qui se met en place à Moscou, en collaboration avec la Chine à l'intérieur du Groupe de Shanghai. De plus, il ne faut pas minimiser les réactions locales face à la politique d'aide au développement dans un contexte croissant de rejet de celle-ci et d'une tentation de rapprochement avec la Russie et la Chine.

L'idée de complot est en grande partie véhiculée en Russie par des intellectuels qui ont une vision singulière de l'Histoire, selon laquelle les processus sociaux sont forcément dus à des manipulations secrètes (Taguieff, 2005, p. 20). Généralement, les théories conspirationnistes servent à penser le recul de quelque chose ${ }^{5}$. Dans le cas russe, l'idée de complot permet de penser le recul de l'influence russe dans son étranger proche et de l'expliquer de manière simpliste. Mais elle manifeste autant une peur, une forme d'incertitude, de doute dans des sociétés postsoviétiques qui

5. Cela peut être celui de l'homme sur terre (extraterrestre), de la chrétienté (complot maçonnique). 
ont connu tant de bouleversements depuis la dislocation de l'URSS. L'analyse du recours à la théorie conspirationniste s'avère nécessaire pour appréhender le fonctionnement des conseillers politiques, notamment russes, qui mettent en place actuellement un nouveau dispositif de pouvoir pour contenir les influences extérieures ( $c f$. compte rendu de l'ouvrage de Pavlovsky). En valorisant le national, ces ripostes peuvent alors prendre des contours nationalistes et chauvins préoccupants.

Il apparaît que, bien au-delà de ces révolutions colorées, ce qui se joue est bien une redéfinition du rôle de l'État, de la souveraineté dans ces espaces politiques. Ces sociétés font face à des contraintes extérieures importantes. Les grandes puissances comme les USA d'un côté, la Chine, la Russie de l'autre, mais aussi les pays musulmans avec leurs fondations caritatives, entendent aussi véhiculer leurs techniques d'influence pour modifier les rapports sociaux. Les États n'ont toutefois pas le monopole de l'influence et certaines institutions (ONG, fondations) ou organisations internationales jouent un rôle prépondérant. Une question se pose alors : jusqu'à quand va-t-on assister à un jeu aussi ouvert ?

On constate néanmoins un paradoxe dans la promotion de la démocratie : l'intervention extérieure favorise le sentiment d'une opinion grandissante qui considère que le choix politique relève de moins en moins d'un choix local et de plus en plus de prises de décision de la part d'experts internationaux et d'institutions lointaines (Union européenne, Banque mondiale, etc.). On peut alors se demander si la multiplication des dispositifs d'assistance à la démocratie ne risque pas de compromettre l'idée de souveraineté populaire et l'instauration de sociétés citoyennes.

D'un point de vue analytique, on peut considérer que les différents programmes de promotion de la démocratie favorisent l'universalisation de l'assistance à la démocratie, qui est un dispositif politique mondialisé, sans obligatoirement contribuer à l'universalisation de la démocratie. Mais ce processus dépasse le simple espace postsoviétique...

\section{Bibliographie}

ABÉLÈs M. (2008), Anthropologie de la globalisation, Fayard, Paris.

AFFANASIEVA E. (2005), Andijanski scenari (Le scénario d'Andijan), Evropa, Moscou.

APPADURAI A. (2001), Après le colonialisme: les conséquences culturelles de la globalisation, Payot, Paris.

BURAWOY M., VERDERY K. (1999), Uncertain Transition: Ethnographies of Change in the Post-socialist World, Boulder, New York-Oxford.

Milani C. (1999), «Globalisation, les organisations internationales et le débat sur la gouvernance» in GratalouP M., Mondialisation: les mots et les choses, Karthala, Paris. 
HÉRODOTE

DeZAlay Y., GARTH B. (2004), «Droits de l'homme et philanthropie hégémonique », Actes de la recherche en sciences sociales, $\mathrm{n}^{\circ} 151-152$.

FERguson J. (1990), The Anti Politics Machine: Development, Depolitization and Bureaucratic Power in Lesotho, Cambridge.

FUKUYAMA F. (1992), The End of history and the last Mann, Hamish, Londres-Hamilton.

GiRARD R. (1972), La Violence et le Sacré, Pluriel, Paris.

GuILHOT N. (2004), «Une vocation philanthropique, George Soros, les sciences sociales et la régulation du marché mondial », Actes de la recherche en sciences sociales, mars.

Guilhot N. (2005), The Democracy Makers: Human Rights and International Order, Columbia University Press, New York.

KILHANI S. (2001), «La société civile, une résurgence », Critique internationale, $\mathrm{n}^{\circ} 10$, janvier.

Koselleck R. (1990), Le Futur passé, EHESS, Paris.

LEFORT C. (1986), Essai sur le politique (XIX et XX $X^{e}$ siècles), Le Seuil, Paris.

HANn C. (1996), Civil Society : Challenging of Western Models, Routledge, Londres.

-(2002), Post-socialism: Ideals, Ideologies and Practices in Eurasia, Routledge, Londres.

Huntington S. (1991), The Third Wave : Democratization in the Late Twentieth Century, University of Oklahoma Press, Norman.

KeAne J. (1998), Civil Society: Old Images, New Visions, Stanford University Press, California.

KNAZEV A. (2006), «Gosudarstvennii perevorot» : 24 marta 2005 v Kirgizii, Bichkek.

MCFALL M. (2002), « The Fourth Wave of Democracy and Dictatorship », World Politics, $\mathrm{n}^{\circ} 54,2$, p. $212-244$.

MARIĆ A. et KRIVOKAPIC M. (2005), «Les faiseurs de révolutions », Politique internationale, $\mathrm{n}^{\circ} 106$.

NyE J. (2004), Soft Power: The Means to Success in World Politics, Public Affairs Press, New York.

PAVlovsKy G. (2005), Kyrgyzsky Perevarot (Le Renversement kirghize), Europa, Moscou.

PECH T. et PADIS M.-O. (2004), Les Multinationales du cœur: les ONG, la politique et le marché, Le Seuil, Paris.

REY A. (1989), Révolution : histoire d'un mot, Gallimard, Paris.

Robinson W. I. (1996), "Globalization, the World System, and Democracy in the US Foreign Policy » in Theory and Society, vol. 25.

SKocPol T. (1985), États et révolutions sociales. La Révolution en France, en Chine, en Russie, Fayard, coll. «Espace du politique», Paris.

Sharp G. (2002), From Dictatorship to Democracy: a Conceptual Framework for Liberation, Albert Einstein Institute, Boston.

TAGUIEFF P.-A. (2005), La Foire aux illuminés: ésotérisme, théorie du complot, extrémisme, Mille et Une Nuits, Paris.

WILSON A. (2006), «Ukraine's Revolution, NGO and the Role of the West », Cambridge Review of International Affairs, vol. XIX, $\mathrm{n}^{\circ} 1$, mars. 